\title{
DERIVATIVES OF BERNSTEIN POLYNOMIALS AND SMOOTHNESS
}

\author{
Z. DITZIAN ${ }^{1}$
}

\begin{abstract}
Equivalence relations between the asymptotic behaviour of derivatives of Bernstein polynomials and the smoothness of the function they approximate are given. This is achieved with an a priori condition that the function is of class $\operatorname{Lip} \beta$ with some small $\beta>0$. The a priori condition is dropped when a similar equivalence relation using the Katorovich operator is proved.
\end{abstract}

1. Introduction. In many articles the rate of convergence of the Bernstein polynomial $B_{n}(f, x)$ to $f(x)$ is related to the smoothness of $f$ (for example, see [1-5]). Sometimes it is possible to find information about the smoothness of $f$ from derivatives of the approximation process, as is well known in the case $P_{y} * f$, where $P_{y}$ is the Poisson kernel and was investigated in many other approximation processes given by convolutions. In this paper the relation will be investigated for the special but important operator of Bernstein polynomials that obviously is not a convolution. We will show that $\left|\Delta_{h}^{2} f\right| \leqslant M h^{\alpha}$ implies

$$
\left|B_{n}^{\prime \prime}(f, t)\right| \leqslant M_{1}\left\{\min \left(n^{2}, n / t(1-t)\right)\right\}^{1-\alpha / 2}
$$

and the latter together with $\left|\Delta_{h}^{2} f\right| \leqslant M_{2} h^{\beta}$ (no matter how small $\beta$ is) implies $\left|\Delta_{h}^{2} f\right| \leqslant M h^{\alpha}$. The situation will be shown to be better for the Kantorovich operator.

2. The main result. The Bernstein polynomials are given by

$$
B_{n}(f, x) \equiv \sum_{k=0}^{n}\left(\begin{array}{l}
n \\
k
\end{array}\right) x^{k}(1-x)^{n-k} f\left(\frac{k}{n}\right) \equiv \sum_{k=0}^{n} f\left(\frac{k}{n}\right) P_{k, n}(x) .
$$

Recalling $\Delta_{h} f(x)=f(x+h)-f(x)$ and $\Delta_{h}^{r} f(x)=\Delta_{h}\left(\Delta_{h}^{r-1} f(x)\right)$, the moduli of continuity are given by

$$
\omega_{r}(f, \eta,[a, b])=\operatorname{Sup}\left\{\left|\Delta_{h}^{r} f(x)\right| ; h \leqslant \eta,[x, x+r h] \subset[a, b]\right\} .
$$

We can now state our result.

THEOREM 2.1. For $f \in C[0,1] \equiv C(I)$ and for some $\beta>0$ and $\omega_{1}(f, \eta, I) \leqslant K \eta^{\beta}$ we have the following equivalence relations:

(a) For $\alpha \leqslant 1, \omega_{1}(f, h, I) \leqslant M h^{\alpha}$ if and only if

$$
\left|B_{n}^{\prime}(f, t)\right| \leqslant M_{1}\left\{\min \left(n^{2}, n / t(1-t)\right)\right\}^{(1-\alpha) / 2} .
$$

Received by the editors April 25, 1983 and, in revised form, October 17, 1983.

1980 Mathematics Subject Classification. Primary 41A17, 41A27.

Key words and phrases. Bernstein polynomial, moduli of smoothness.

${ }^{1}$ Supported by NSERC grant A4816 of Canada. 
(b) For $\alpha \leqslant 2, \omega_{2}(f, h, I) \leqslant M h^{\alpha}$ if and only if

$$
\left|B_{n}^{\prime \prime}(f, t)\right| \leqslant M_{1}\left\{\min \left(n^{2}, n / t(1-t)\right)\right\}^{1-\alpha / 2} \text {. }
$$

REMARK. Obviously if $\omega_{i}(f, h, I) \leqslant M h^{\alpha}(i \geqslant 1)$, the condition $\left|\Delta_{h} f(x)\right| \leqslant K h^{\beta}$ is redundant. We will later show that in some cases this additional side condition can be removed. It is an open question whether it can always be removed.

For our proof we will need the following lemma:

LEMMA 2.2. For $f \in C[0,1]$ there exist $f_{1, \eta}$ and $f_{2, \eta}$ such that

$$
\begin{gathered}
\left|f(x)-f_{1, \eta}(x)\right| \leqslant \omega_{1}(f, \eta / 2), \quad\left|f(x)-f_{2, \eta}(x)\right| \leqslant 5 \omega_{2}(f, \eta), \\
\left|f_{1, \eta}^{\prime}(x)\right| \leqslant \frac{1}{\eta} \omega_{1}(f, \eta) \quad \text { and } \quad\left|f_{2, \eta}^{\prime \prime}(x)\right| \leqslant \frac{5}{\eta^{2}} \omega_{2}(f, \eta) .
\end{gathered}
$$

Proof. We can extend $f$ to $R$ such that $\omega_{1}(f, \eta, R)=\omega_{1}(f, \eta, I)$ and $\omega_{2}(f, \eta, R)$ $\leqslant 5 \omega_{2}(f, \eta, I)$ (see Timan [6, p. 122]). We define for the new function $f$ (differently for $i=1,2$, outside of $I$ ) the usual Stekelov functions $f_{1, \eta}(x)$ and $f_{2, \eta}(x)$ for all $x$ (2.3)

$$
f_{1, \eta}(x)=\frac{1}{h} \int_{-h / 2}^{h / 2} f(x+u) d u, \quad f_{2, \eta}(x)=\frac{1}{h^{2}} \int_{-h / 2}^{h / 2} \int_{-h / 2}^{h / 2} f(x+u+v) d u d v
$$

and the result follows from [6, p. 163] and the extension theorem mentioned above.

Proof of The Theorem 2.1. We first deal with the easy implication, that is, $\omega_{i}(f, h, I) \leqslant M h^{\alpha}$ for $i=1,2$ implies

Recalling

$$
\left|B_{n}^{(i)}(f ; t)\right| \leqslant M_{1}(\min \{n, \sqrt{n / t(1-t)}\})^{i-\alpha} .
$$

and

$$
B_{n}^{\prime}(f, t)=n \sum_{k=0}^{n-1} \Delta_{1 / n} f\left(\frac{k}{n}\right) P_{k, n-1}(t)
$$

$$
B_{n}^{\prime \prime}(f, t)=n(n-1) \sum_{k=0}^{n-2} \Delta_{1 / n}^{2} f\left(\frac{k}{n}\right) P_{k, n-2}(t),
$$

we have $\left|B_{n}^{\prime}(f, t)\right| \leqslant n \omega_{1}(f, 1 / n, I) \leqslant M n^{1-\alpha}$ and $\left|B_{n}^{\prime \prime}(f, t)\right| \leqslant n^{2} \omega_{2}(f, 1 / n, I) \leqslant$ $M n^{2-\alpha}$. Using $\left|B_{n}^{\prime \prime}(f, t)\right| \leqslant 5 \omega_{2}(f, \eta, I)\left[n / t(1-t)+\eta^{-2}\right]$ (see Becker [2, Lemma 1]) and choosing $\eta=\sqrt{t(1-t) / n}$, we have $\left|B_{n}^{\prime \prime}(f, t)\right| \leqslant 10 M(n / t(1-t))^{(2-\alpha) / 2}$ which completes the estimate of $\left|B_{n}^{\prime \prime}(f, t)\right|$ (with $M_{1}=10 M$ ). Similarly (using Lemma 2.2), we write

$$
\begin{aligned}
\left|B_{n}^{\prime}(f, t)\right| \leqslant & \left|B_{n}^{\prime}\left(f-f_{1, \eta}, t\right)\right|+\left|B_{n}^{\prime}\left(f_{1, \eta}, t\right)\right| \\
\leqslant & \left\|f-f_{1, \eta}\right\|_{C[0,1]} \frac{1}{t(1-t)} \sum_{k=0}^{n}|k-n t| P_{k, n}(t) \\
& +\left|n \sum_{k=0}^{n-1} \Delta_{1 / n} f_{1, \eta}\left(\frac{k}{n}\right) P_{k, n-1}(t)\right| \\
\leqslant & \omega_{1}(f, \eta, I)\left(\frac{n}{t(1-t)}\right)^{1 / 2}+\sum_{k=0}^{n-1}\left|f_{1, \eta}^{\prime}\left(\xi_{k}\right)\right| P_{k, n-1}(t) \\
\leqslant & \omega_{1}(f, \eta, I)\left(\frac{n}{t(1-t)}\right)^{1 / 2}+\frac{1}{\eta} \omega_{1}(f, \eta, I),
\end{aligned}
$$


and choosing $\eta=\sqrt{t(1-t) / n}$, we have $\mid B_{n}^{\prime}(f, t) \leqslant 2 M(\sqrt{n / t(1-t)})^{1-\alpha}\left(M_{1}=\right.$ $2 M$ for (a)).

To prove the if part (of both (a) and (b)), we now write

and similarly,

$$
\begin{aligned}
\left|\Delta_{h} f(x)\right| & \leqslant\left|\Delta_{h}\left\{f(x)-B_{n}(f, x)\right\}\right|+\left|\int_{x}^{x+h} B_{n}^{\prime}(f, u) d u\right| \\
& \leqslant 2 \max _{u=x, x+h}\left|f(u)-B_{n}(f, u)\right|+\int_{x}^{x+h}\left|B_{n}^{\prime}(f, u)\right| d u,
\end{aligned}
$$

$$
\begin{aligned}
\left|\Delta_{h}^{2} f(x)\right| \leqslant & \left|\Delta_{h}^{2}\left\{f(x)-B_{n}(f, x)\right\}\right|+\left|\Delta_{h}^{2} B_{n}(f, x) d u\right| \\
\leqslant & 4 \max _{u=x, x+h, x+2 h}\left|f(u)-B_{n}(f, u)\right|+\left|\int_{x}^{x+h}(v-x) B_{n}^{\prime \prime}(f, v) d v\right| \\
& +\left|\int_{x+h}^{x+2 h}(v-x-2 h) B_{n}^{\prime \prime}(f, v) d v\right| .
\end{aligned}
$$

Using, $f_{i, \eta}$ defined in Lemma 2.2, writing $f=f-f_{i, \eta}+f_{i, \eta}$ and substituting in $\left|f(u)-B_{n}(f, u)\right|$, we obtain $\left|f(u)-B_{n}(f, u)\right| \leqslant 15 \omega_{2}(f, \sqrt{u(1-u) / n})$ as was done by Strukov and Timan [5], and similarly, $\left|f(u)-B_{n}(f, u)\right| \leqslant 3 \omega_{1}(f, \sqrt{u(1-u) / n})$. (We can deduce the second inequality with a somewhat worse constant from the first inequality but direct use of the method yields the constant 3.)

Using the estimates above for $\Delta_{h} f(x)$ and $\Delta_{h}^{2} f(x)$ as well as the estimates for $\left|f(u)-B_{n}(f, u)\right|$, we now obtain

$$
\left|\Delta_{h} f(x)\right| \leqslant 6 \omega_{1}\left(f, \delta_{1}(n, x, h)\right)+\int_{x}^{x+h}\left|B_{n}^{\prime}(f, u)\right| d u,
$$

where $\delta_{1}(n, x, h)=\max _{u=x, x+h} \sqrt{u(1-u) / n}$, and

$$
\begin{aligned}
\left|\Delta_{h}^{2} f(x)\right| \leqslant & 4 \cdot 15 \omega_{2}\left(f, \delta_{2}(n, x, h)\right)+\left|\int_{x}^{x+h}(v-x) B_{n}^{\prime \prime}(f, v) d v\right| \\
& +\left|\int_{x+h}^{x+2 h}(v-x-2 h) B_{n}^{\prime \prime}(f, v) d v\right|,
\end{aligned}
$$

where $\delta_{2}(n, x, h)=\max _{u=x, x+h, x+2 h} \sqrt{u(1-u) / n}$. Without loss of generality we restrict $h$ by $h<\frac{1}{8}$. We estimate $\Delta_{h} f$ now. Using $\left|B_{n}^{\prime}(f, t)\right| \leqslant M_{1} n^{1-\alpha}$, we have $\int_{x}^{x+h}\left|B_{n}^{\prime}(f, u)\right| d u \leqslant M_{1} h n^{1-\alpha}$. Using $\left|B_{n}^{\prime}(f, t)\right| \leqslant M_{1}(n / t(1-t))^{(1-\alpha) / 2}$ in the range $0 \leqslant x \leqslant 1-x-h$, we have, for $x \geqslant h$,

$$
\begin{aligned}
\int_{x}^{x+h}\left|B_{n}^{\prime}(f, t)\right| d t & \leqslant M_{1} h\left(\frac{n}{x(1-x)}\right)^{(1-\alpha) / 2} \leqslant 2 M_{1} h\left(\frac{n}{2 x(1-\ddot{x})}\right)^{(1-\alpha) / 2} \\
& \leqslant 2 M_{1} h\left(\frac{n}{(x+h)(1-x-h)}\right)^{(1-\alpha) / 2}
\end{aligned}
$$

and, for $x<h$,

$$
\begin{aligned}
\int_{x}^{x+h}\left|B_{n}^{\prime}(f, t)\right| d t & \leqslant M_{1} \int_{0}^{x+h}\left(\frac{n}{t(1-t)}\right)^{(1-\alpha) / 2} \\
& <\frac{4}{3} M_{1} n^{(1-\alpha) / 2} \frac{2}{(\alpha+1)}(x+h)^{(\alpha+1) / 2} \\
& \leqslant \frac{8}{3} M_{1} n^{(1-\alpha) / 2}(2 h)\left(\frac{1}{(x+h)(1-x-h)}\right)^{(1-\alpha) / 2} \\
& \leqslant \frac{16}{3} M_{1} h\left(\frac{n}{(x+h)(1-x-h)}\right)^{(1-\alpha) / 2}
\end{aligned}
$$


For $1-x \leqslant x+h(1-x-h \leqslant x)$ the roles of $x$ and $1-x$ and that of $x$ and $x+h$ in the proof are reversed and together with the above we have

$$
\int_{x}^{x+h}\left|B_{n}^{\prime}(f, u)\right| d u \leqslant 6 M_{1} h(\min \{n, \sqrt{n / x(1-x)}, \sqrt{n /(x+h)(1-x-h)}\})^{1-\alpha} .
$$

We can now choose $n$ big enough (for the given $x$ and $h$ ) such that, $\delta_{1}(n, x, h) \leqslant$ $h / T_{1}$ while $\min \{n, \sqrt{n / x(1-x)}, \sqrt{n /(x+h)(1-x-h)}\} \leqslant 2 T_{1} / h$ for $T_{1}>1$ which will satisfy $T_{1}^{\beta}>12$. This is possible since for $x(1-x) \leqslant(x+h)(1-x-h)$ we choose the smallest $n, n \geqslant 1$, for which

$$
\delta(n, x, h)=\sqrt{\frac{(x+h) / 1-x-h}{n}}<\frac{h}{T_{1}} ;
$$

if $n=1$ we use $1 \leqslant 2 T_{1} / h$, otherwise

$$
\begin{aligned}
& \min \{n, \sqrt{n / x(1-x)}, \sqrt{n /(x+h)(1-x-h)}\} \\
& \leqslant \sqrt{n /(x+h)(1-x-h)} \\
& \leqslant \sqrt{2} \sqrt{(n-1) /(x+h)(1-x-h)} \leqslant \sqrt{2} T_{1} / h \leqslant 2 T_{1} / h .
\end{aligned}
$$

For $x(1-x) \geqslant(x+h)(1-x-h)$ take $x$ instead of $x+h$, and $1-x$ instead of $x$.

Combining the estimate of $\Delta_{h} f(x)$ and the choice of $n$, we have

$$
\begin{aligned}
\left|\Delta_{h} f(x)\right| & \leqslant 6 \omega_{1}\left(f, h / T_{1}\right)+6 M_{1} h\left(2 T_{1} / h\right)^{1-\alpha} \\
& \equiv A_{1} \omega_{1}\left(f, h / T_{1}\right)+B_{1} h\left(T_{1} / h\right)^{1-\alpha}
\end{aligned}
$$

Note now that in (2.4) the right side does not depend on $x$ nor on $B_{n}(f, t)$.

We now proceed with the estimate of $\Delta_{h}^{2} f(x)$ for $0<h<\frac{1}{8}$. Using $\left|B_{n}^{\prime \prime}(f, t)\right| \leqslant$ $M_{1} n^{2-\alpha}$, we have

$$
\int_{x}^{x+h}(v-x)\left|B_{n}^{\prime \prime}(f, v)\right| d v \leqslant M_{1} h^{2} n^{2-\alpha} / 2
$$

and

$$
\int_{x+h}^{x+2 h}|(v-x-2 h)|\left|B_{n}^{\prime \prime}(f, v)\right| d v \leqslant M_{1} h^{2} n^{2-\alpha} / 2 .
$$

For $x \leqslant 1-x-2 h$, and therefore

$$
(x+2 h)(1-x+2 h) \geqslant x(1-x)
$$

(but $(x+h)(1-x-h)$ may be bigger than either), we have, for $x \geqslant h$,

$$
\begin{aligned}
& \int_{x}^{x+h}(v-x)\left|B_{n}^{\prime \prime}(f, v)\right| d v \leqslant M_{1} h^{2}\left(\frac{n}{x(1-x)}\right)^{1-\alpha / 2} \frac{1}{2} \leqslant \frac{3}{2} M_{1} h^{2}\left(\frac{n}{3 x(1-x)}\right)^{1-\alpha / 2} \\
& \leqslant M_{1} \frac{3}{2} h^{2}\left(\frac{n}{(x+2 h)(1-x-2 h)}\right)^{1-\alpha / 2}
\end{aligned}
$$

and similarly

$$
M_{1} h^{2}\left(\frac{n}{x(1-x)}\right)^{1-\alpha / 2} \frac{1}{2} \leqslant M_{1} h^{2}\left(\frac{n}{(x+h)(1-x-h)}\right)^{1-\alpha / 2} .
$$


We also have (still for $x \leqslant 1-x-2 h$ ), for $x \leqslant h$,

$$
\begin{gathered}
\int_{x}^{x+h}(v-x)\left|B_{n}^{\prime \prime}(f, v)\right| d v \leqslant M_{1} n^{1-\alpha / 2} \int_{x}^{x+h}(v-x)(v(1-v))^{-1+\alpha / 2} d v \\
\leqslant M_{1} n^{1-\alpha / 2} \frac{4}{3} \int_{0}^{x+h} v^{\alpha / 2} d v=M_{1} \frac{4}{3} n^{1-\alpha / 2} \frac{1}{1+\alpha / 2}(x+h)^{1+\alpha / 2} \\
\leqslant M_{1} \frac{4}{3} n^{1-\alpha / 2}(x+2 h)^{1+\alpha / 2} \leqslant M_{1} \frac{4}{3} n^{1-\alpha / 2}(3 h)^{2}(x+2 h)^{-1+\alpha / 2} \\
\leqslant 12 M_{1} h^{2}\left(\frac{n}{(x+2 h)(1-x-2 h)}\right)^{1-\alpha / 2} .
\end{gathered}
$$

The estimate of $\left|\int_{x+h}^{x+2 h}(v-x-2 h)\right| B_{n}^{\prime \prime}(f, v)|d v|$ for $x \leqslant 1-x-2 h$ and the estimate of both terms for $x \geqslant 1-x-2 h$ are similar. (In the latter the roll of $x$ and $x+2 h$ and that of $x$ and $1-x$ are reversed.) We now have

$$
\begin{aligned}
\left|\Delta_{h}^{2} f(x)\right| \leqslant & 60 \omega_{2}\left(f, \delta_{2}(n, x, h)\right) \\
& +M_{1} h^{2} 24(\min (n, \sqrt{n / x(1-x)}, \sqrt{n /(x+h)(1-x-h)}, \\
& \sqrt{n /(x+2 h)(1-x-2 h)}))^{2-\alpha} .
\end{aligned}
$$

Choosing $n$ (in a similar way to the choice made to prove (2.4)) such that $\delta_{2}(n, x, h) \leqslant h / T_{2}$ while

$$
\begin{aligned}
\min (n, \sqrt{n / x(1-x)}, \sqrt{n /(x+h)(1-x-h)}, & \\
& \sqrt{n /(x+2 h)(1-x-2 h)}) \leqslant 2 T_{2} / h
\end{aligned}
$$

for some $T_{2}, T_{2}>1$ (we will choose $T_{2}$ such that $T_{2}^{\beta}>120$ ), we have

$$
\left|\Delta_{h}^{2} f(x)\right| \leqslant A_{2} \omega_{2}\left(f, h / T_{2}\right)+h^{2} B_{2}\left(T_{2} / h\right)^{2-\alpha} \text {, }
$$

where $A_{2}=60$ and $B_{2}=48 M_{1}$.

We will now show that $\left|\Delta_{h} f(x)\right| \leqslant K h^{\beta}$ for some $\beta$ implies $\left|\Delta_{h} f(x)\right| \leqslant M_{2} h^{\alpha}$ and $\left|\Delta_{h}^{2} f(x)\right| \leqslant M_{2} h^{\alpha}$ for (a) and (b) of our theorem. Choosing $T_{1}$ such that $T_{1}^{\beta}>2 A_{1}=$ 12 we write

$$
\begin{aligned}
\left|\Delta_{h} f(x)\right| & \leqslant A_{1} \omega_{1}\left(f, h / T_{1}\right)+B_{1} h\left(T_{1} / h\right)^{1-\alpha} \\
& \leqslant A_{1}^{2} \omega_{1}\left(f, h / T_{1}^{2}\right)+B_{1} T_{1}^{1-\alpha} h^{\alpha}\left(1+A_{1} T_{1}^{-\alpha}\right) \\
& \leqslant A_{1}^{s} \omega_{1}\left(f, h / T_{1}^{s}\right)+B_{1} T_{1}^{1-\alpha} h^{\alpha}\left(1+A_{1} T_{1}^{-\alpha}+\cdots+A_{1}^{s-1} T_{1}^{-(s-1) \alpha}\right) \\
& \leqslant A_{1}^{s} \omega_{1}\left(f, h / T_{1}^{s}\right)+B_{1} T_{1}^{1-\alpha} h^{\alpha}\left(1-A_{1} T_{1}^{-\alpha}\right)^{-1}
\end{aligned}
$$

and since, for $T_{1}^{\beta}>2 A_{1}, A_{1}^{s} \omega_{1}\left(f, h / T_{1}^{s}\right)$ tends to zero, we have $\left|\Delta_{h} f(x)\right| \leqslant$ $B_{1} T_{1}^{1-\alpha}\left(1-A_{1} T_{1}^{-\alpha}\right)^{-1} h^{\alpha}$ which, since $T_{1}^{\alpha}>T_{1}^{\beta}>2 A_{1}$, implies $\left|\Delta_{h} f(x)\right| \leqslant M_{2} h^{\alpha}$. Similarly, for $T_{2}^{\beta}>2 A_{2}$ we have

$$
\begin{aligned}
\left|\Delta_{h}^{2} f(x)\right| & \leqslant A_{2} \omega_{2}\left(f, h / T_{2}\right)+B_{2} h^{2}\left(T_{2} / h\right)^{2-\alpha} \\
& \leqslant A_{2}^{s} \omega_{2}\left(f, h / T_{2}^{s}\right)+B_{2} T_{2}^{2-\alpha}\left(1-A_{2} T_{2}^{-\alpha}\right)^{-1} h^{\alpha}
\end{aligned}
$$

and hence $\left|\Delta_{h}^{2} f(x)\right| \leqslant B_{2} T_{2}^{2-\alpha}\left(1-A_{2} T_{2}^{-\alpha}\right)^{-1} h^{\alpha} \leqslant M_{2} h^{\alpha}$. 
3. Remarks and conclusions. For proving that $f(x)$ belongs to Lipschitz class $\alpha$ we need the a priori condition that $f(x)$ satisfies Lipschitz conditon of order $\beta$ (no matter how small). This a priori condition is not desirable and it seems interesting to find whether it can be dropped. For the case below it will be shown that it can be.

THEOREM 3.1. For $f \in C[0,1],\left|B_{n}^{\prime}(f, x)\right| \leqslant M_{1}\left\{\min \left(n^{2}, n / x(1-x)\right)\right\}^{(1-\alpha) / 2}$ and $f$ monotonic imply $\left|\Delta_{h} f(x)\right| \leqslant M h^{\alpha}$.

Proof. For $f$ monotonic $\Delta_{1 / n} f(k / n)$ is of fixed sign, and therefore,

$$
\left|B_{n}^{\prime}(f, x)\right|=n \sum_{k=0}^{n-1}\left|\Delta_{1 / n} f\left(\frac{k}{n}\right)\right| P_{k, n-1}(x) \leqslant M_{1}\left\{\min \left(n^{2}, n / x(1-x)\right)\right\}^{(1-\alpha) / 2} \text {. }
$$

Using Stirling's estimate for $m$ !, we obtain, for $0<k<n-1$,

$$
P_{k, n-1}\left(\frac{k}{n-1}\right) \geqslant C \cdot \frac{1}{\sqrt{n}((k /(n-1))(1-k /(n-1)))^{1 / 2}}
$$

for some $C>0$, independent of $n$ and $k$, and therefore, for those $k$ using $x=$ $k /(n-1)$,

$$
\left|B_{n}^{\prime}\left(f, \frac{k}{n-1}\right)\right| \geqslant n\left|\Delta_{1 / n} f\left(\frac{k}{n}\right)\right| P_{k, n-1}\left(\frac{k}{n-1}\right)
$$

and therefore

$$
\left|\Delta_{1 / n} f\left(\frac{k}{n}\right)\right| \leqslant \frac{M_{1}}{C}\left(\frac{(k /(n-1))(1-k /(n-1))}{n}\right)^{\alpha / 2} \leqslant \frac{M_{1}}{C} n^{-\alpha / 2} .
$$

For $x=0$ and $x=1$ we have $\left|\Delta_{1 / n} f(0)\right| \leqslant M_{1} n^{-\alpha}$ and $\left|\Delta_{1 / n}((n-1) / n)\right| \leqslant M n^{-\alpha}$.

Therefore Theorem 2.1 implies that $f \in \operatorname{Lip} \alpha$ using $\left|\Delta_{1 / n} f(k / n)\right| \leqslant K n^{-\alpha / 2}$ for all $k$ and $n$, or $f \in \operatorname{Lip}(\alpha / 2)(\beta=\alpha / 2)$.

A more interesting application of Theorem 2.1 is the estimate for the Kantorovich modification of Bernstein polynomials. The Kantorovich operator is given by

$$
\begin{aligned}
K_{n}(f, t) & =\sum_{k=0}^{n}\left(\begin{array}{l}
n \\
k
\end{array}\right) t^{k}(1-t)^{n-k}(n+1) \int_{k / n+1}^{(k+1) / n+1} f(u) d u \\
& =\frac{d}{d t} B_{n+1}(F, t),
\end{aligned}
$$

where $F(x)=\int_{0}^{x} f(u) d u$.

TheOREM 3.2. For $f \in C[0,1]$ and $\alpha \leqslant 1$ we have $\left|\Delta_{h} f(x)\right| \leqslant M h^{\alpha}$ if and only if $\left|K_{n}^{\prime}(f, t)\right| \leqslant M_{1}(\min (n, \sqrt{n / x(1-x)}))^{1-\alpha}$.

REMARK. We observe that here a condition $f \in \operatorname{Lip} \beta$ is not required.

Proof. We observe $\left|\Delta_{h} f\right| \leqslant M h^{\alpha}$ implies $\left|\Delta_{h}^{2} F(x)\right| \leqslant M h^{1+\alpha}$ and therefore

$$
\left|B_{n+1}^{\prime \prime}(F, x)\right|=\left|K_{n}^{\prime}(f, x)\right| \leqslant M_{1}(\min (n+1, \sqrt{n+1 / x(1-x)}))^{1-\alpha} .
$$

Assume now that

$$
\left|K_{n}^{\prime}(f, x)\right|=\left|B_{n+1}^{\prime \prime}(F, x)\right| \leqslant M(\min (n, \sqrt{n / x(1-x)}))^{2-(1+\alpha)} ;
$$


this implies for $F(x) \in \operatorname{Lip} 1$, which follows $\left|\Delta_{h} F(x)\right| \leqslant h\|f\|$, that $\left|\Delta_{h}^{2} F(x)\right| \leqslant$ $M_{1} h^{1+\alpha}$ and this implies $\left|\Delta_{h} f(x)\right| \leqslant M_{1} h^{\alpha}$.

Actually the following even "more general" statement is valid.

TheOREM 3.3. For $f \in L_{p}[0,1], p>1$, and $\alpha \leqslant 1$ the condition $\left|\Delta_{h} f(x)\right| \leqslant M h^{\alpha}$ is equivalent to $\left|K_{n}^{\prime}(f, t)\right| \leqslant M_{1}(\min (n, \sqrt{n / x(1-x)}))^{1-\alpha}$.

Note that though the initial condition is $f \in L_{p}[0,1]$, we show that $f$ is a continuous function. The proof follows the observation that $F(x)=\int_{0}^{x} f(u) d u$, and therefore

$$
\begin{aligned}
|F(x+h)-F(x)| & =\left|\int_{x}^{x+h} f(u)\right| d u \leqslant\left\{\int_{x}^{x+h} 1\right\}^{1 / q}\left\{\int_{x}^{x+h}|f(u)|^{p} d u\right\}^{1 / p} \\
& \leqslant h^{1 / q}\|f\|_{L_{p}}
\end{aligned}
$$

where $1 / q+1 / p=1$. Now $F(x) \in \operatorname{Lip} q^{-1}$ and satisfies the condition on $f$ in Theorem 2.1 which completes the proof, recalling $K_{n}^{\prime}(f, t)=B_{n}^{\prime \prime}(F, t)$. (Of course we have just shown that $f$ is equivalent to a function in $C[0,1]$ satisfying Lip* $\alpha$ condition.)

\section{REFERENCES}

1. H. Berens and G. G. Lorentz, Inverse theorems for Bernstein polynomials, Indiana Univ. Math. J. 21 (1976), 693-708.

2. M. Becker, An elementary proof of the inverse theorem for Bernstein polynomials, Aequationes Math. 19 (1979), 145-150.

3. Z. Ditzian, A global inverse theorem for combinations of Bernstein polynomials, J. Approx. Theory 26 (1979), 277-292.

4. Interpolation and the rate of convergence of Bernstein polynomials, Approximation Theory. III (W. Cheney, ed.), Academic Press, New York, 1980, pp. 241-347.

5. L. I. Strukov and A. F. Timan, Mathematical expectation of continuous functions of random variables. Smoothness and variance, Siberian Math. J. 18 (1978), 469-479.

6. A. F. Timan, Theory of approximation of functions of a real variable, English transl., Macmillan, New York, 1963.

Department of Mathematics, University of Alberta, Edmonton, Alberta, Canada T6G 2G1 